\title{
Dental Adhesion Enhancement on Zirconia Inspired by Mussel's Priming Strategy Using Catechol
}

\author{
Minsu Park ${ }^{1}$, Sungwon Ju ${ }^{1,2}$, Roscoe Linstadt ${ }^{2,3}$, Jinsoo Ahn ${ }^{1, *}$ and Kollbe Ahn ${ }^{2,3, *(1)}$ \\ 1 Dental Biomaterials and Dental Research Institute, Seoul National University, Seoul 03080, Korea; \\ bimmer740@naver.com (M.P.); epoxy609@naver.com (S.J.) \\ 2 Marine Science Institute, University of California, Santa Barbara, CA 93106, USA; rlinstadt1988@gmail.com \\ 3 Department of Chemistry, University of Central Florida, Orlando, FL 32816, USA \\ * Correspondence: ahnjin@snu.ac.kr (J.A.); kollbe@ucf.edu (K.A.); Tel.: +1-407-823-2973 (K.A.)
}

Received: 9 August 2018; Accepted: 21 August 2018; Published: 24 August 2018

\begin{abstract}
Zirconia has recently become one of the most popular dental materials in prosthodontics being used in crowns, bridges, and implants. However, weak bonding strength of dental adhesives and resins to zirconia surface has been a grand challenge in dentistry, thus finding a better adhesion to zirconia is urgently required. Marine sessile organisms such as mussels use a unique priming strategy to produce a strong bonding to wet mineral surfaces; one of the distinctive chemical features in the mussel's adhesive primer proteins is high catechol contents among others. In this study, we pursued a bioinspired adhesion strategy, using a synthetic catechol primer applied to dental zirconia surfaces to study the effect of catecholic priming to shear bond strength. Catechol priming provided a statistically significant enhancement $(p<0.05)$ in shear bond strength compared to the bonding strength without priming, and relatively stronger bonding than commercially available zirconia priming techniques. This new bioinspired dental priming approach can be an excellent addition to the practitioner's toolkit to improve dental bonding to zirconia.
\end{abstract}

Keywords: zirconia; primer; priming; bonding; catechol; dental; prosthodontics

\section{Introduction}

Ceramics have been widely used for dental and biomaterials, e.g., for prostheses. Zirconia has recently become one of the most popular ceramic dental and biomaterials with its superior mechanical properties: highly tough and strong, wear resistant, shock resistant, while both chemically and dimensionally stable [1]. Its superior mechanical properties are due to a martensitic type phase transformation that prevents crack propagation [2]. However, owing to its high chemical resistance, traditional dental ceramic bonding techniques (e.g., chemical ceramic etching) are not compatible with zirconia [2]. Traditional mechanical grinding or sand blasting methods on dental ceramics can be an alternative, but it may cause the phase transformation and hydrothermal fatigue degradation [3].

Priming (or adhesive priming) is a surface treatment that can promote adhesion of coatings or adhesives to the substrate of interest. Silane-based primers (known as silane coupling agents) are commonly used to prime dental surfaces, including ceramics. Despite many attempts to improve the bonding strength to zirconia using silane-based coupling agents, no significant improvement has been reported yet [3].

In the past decades, the National Institute of Dental and Craniofacial Research (NIDCR) has supported interdisciplinary studies such as marine mussel adhesion as the oral environment has much in common with the intertidal environment; both oral and marine adhesions are subjected to opportunistic microbes; cyclic stress; constant fluid flow; and variations in salinity, temperature, and $\mathrm{pH}$ [4]. Figure 1 shows marine sessile organisms adhere to rocks at the seashore. The NIDCR's 
long-term investment to the bioadhesion $[4,5]$ has recently begun to show potential for practical dental [6] and biomedical [7,8] applications. Several dental applications of catechol have been reported using catechol-containing polymers such as tannin as a dental sealant [9] and catecholic methacrylamide for a dental adhesive [10] via catechol-ferric iron complexation. Despite the inherent aesthetic limitations of Fe-catechol complexation, (complexes are highly colored and darken over time) the previous work exposed the potential of catechol chemistry for dental materials applications. In contrast to these previous approaches which mimic the mussels' use of catecholic complexation and crosslinking chemistry for enhancement of cohesive strength within the byssus, we, in this study instead aimed to mimic the mussel's means of priming surfaces with catechol-mediated hydrogen bonding that increases the adhesive strength of mussel plaque to mineral surfaces [11].

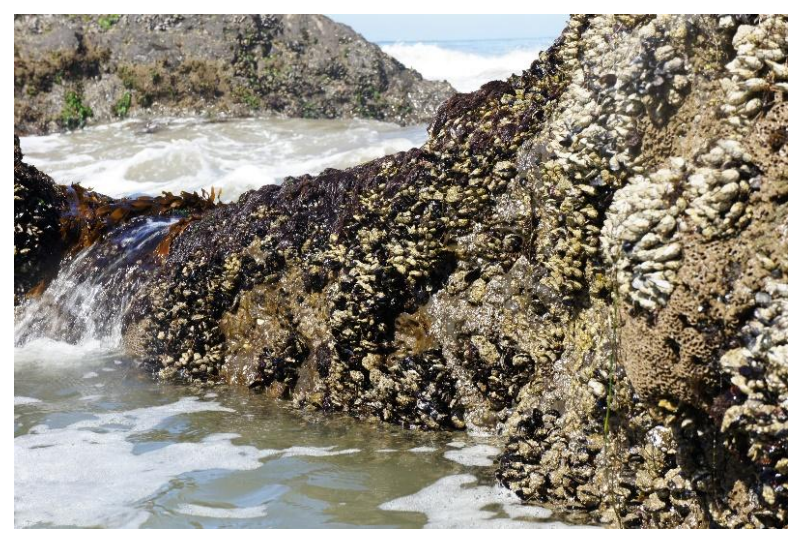

Figure 1. Mussels and other marine sessile organisms such as mussels anchored to mineral surfaces at seashore in Santa Barbara, CA, USA.

One of the useful chemical features of mussels' adhesive mechanism is the use of catechol- and phospho-rich proteins as surface primers [11-13]. Similarly, in dentistry phosphorous-based primers such as 10-methacryloyloxydecyl dihydrogen phosphate (MDP) are commonly used based in part on the strength of phosphate binding to calcium ions in hydroxyapatite (the main mineral in human teeth-higher than $90 \mathrm{wt} \%$ in enamel and $70 \mathrm{wt} \%$ in dentin-and in human bone-up to $70 \mathrm{wt} \%$ ). By bridging the surface minerals to a polymerizable methacrylate, MDP helps to achieve bonding performance of methacrylate-based dental resins.

MDP has also been shown to improve adhesion of dental resins to zirconia surface by ionic phosphate-Zr binding [3,14], yet further increases to the strength of such resins bound to zirconia is urgently required and many researchers are looking for alternative chemistry that can fulfill this demand. Very recently, our group has reported adhesion enhancement of dental adhesion to silica, steel, and tooth enamel using the catechol-containing primers [6,15]. In contrast to the large body of literature regarding phosphate-primers, and despite over 10,000 peer-reviewed papers published within the past decade on catechol-mediated biological adhesion [11], the use of catechol chemistry for strength enhancement and surface priming in dental settings remains largely unexplored.

In this study, we investigated if one of the most pressing issues in prosthodontics-poor adhesion to zirconia-could be overcome with catecholic bioinspired surface priming. Using a previously reported bifunctional catechol-methacrylate to prime zirconia, we were able to demonstrate significant improvement in the knife shear bond strength compared to untreated surfaces. Moreover, the bonding performance is superior to commercial dental primers reliant on acidic phosphate and carboxylate functionality. Our results highlight the potential of catechol-mediated surface priming to solve bonding issues with zirconia for dental and biomedical applications. In addition, we were able to correlate bonding performance with surface coverage as measured by scanning electron microscope (SEM) and offer an improved synthetic method to access the catechol methacrylate primer in higher purity compared to the previously reported method $[6,16,17]$. 


\section{Materials and Methods}

All chemicals for synthesis were purchased from Sigma-Aldrich (St. Louis, MO, USA). All dental resins and primers were purchased as shown in Table 1.

Table 1. Commercial zirconia dental primers.

\begin{tabular}{ccc}
\hline Primers & Composition $^{\mathbf{1}}$ & Manufacturer \\
\hline Zirconia liner (ZL) & 4-META & SunMedical Co. Ltd (Shiga, Japan) \\
Alloy primer (AP) & 10-MDP, VBATDT & Kuraray Dental, Inc. (Osaka, Japan) \\
Universal primer (UP) & MAC-10, MTU-6 & Tokuyama Dental, Corp. (Tokyo, Japan) \\
\hline
\end{tabular}

${ }^{1}$ 4-META: 4-methacryloyloxy ethyl trimellitate anhydride; 10-MDP: 10-Methacryloyloxydecyl dihydrogen phosphate; VBATDT: 6-(4-Vinylbenzyl-n-propyl)amino-1,3,5-triazine-2,4-dithiol; MAC-10: 11-Methacryloxyundecane-1,1-dicarboxylic acid; MTU-6: 6-methacryloyloxyhexyl-2-thiouracil-5-carboxylate.

\subsection{Synthesis of Catechol Methacrylate Primer}

Triethylsilyl (TES)-protected (or silylated) catechol-methacrylate was synthesized from eugenol by the previously reported method [16] and generously provided to us by Osaka Organic Chemical Industry, Ltd. (Osaka, Japan). By modifying the previously reported conditions for TES-deprotection, the catecholic methacrylate could be obtained more economically, with a higher degree of purity according to the following procedure. $411 \mathrm{mg}$ of TES-protected catechol methacrylate $(0.855 \mathrm{mmol}$, 1 equiv.) and $229 \mathrm{mg}$ of benzoic acid ( $1.88 \mathrm{mmol}, 2.2$ equiv., 1.1 equiv. per TES group) were dissolved in ca. 3-5 mL of tetrahydrofuran (THF), (note: distilled/anhydrous THF was not used), and stirred at ambient temperature, whereupon a $1 \mathrm{M}$ solution of tetrabutylammonium fluoride (TBAF) in THF (1.88 $\mathrm{mL}, 1.88 \mathrm{mmol}, 2.2$ equiv.) was added dropwise to the stirred mixture. The mixture was stirred at ambient temperature until thin-layer chromatography (TLC) indicates complete conversion (ca. 30-120 min). Once judged complete, the stir bar was removed, and THF was removed by rotary evaporation. The crude residue was then suspended in $150 \mathrm{~mL}$ of ether $\left(\mathrm{Et}_{2} \mathrm{O}\right)$, and washed twice with DI water, once with brine, dried over sodium sulfate. Once dry, the solution was filtered and evaporated under reduced pressure, whereupon the residue was dissolved in a minimum amount of dichloromethane (DCM), loaded on top of a silica column, and purified further by flash chromatography. It should be noted that catechols adhere strongly to silica, and are subject to decomposition during chromatography, thus chromatography was performed quickly, and with a minimum amount of silica gel. Oxidative decomposition during chromatography could be mitigated by addition of a small amount $(0.1 \% v / v)$ of $\mathrm{AcOH}$ to the eluent, although this required prolonged and undesirable amounts of time on high-vacuum to remove traces of acid. The use of silica gel impregnated with ascorbic acid was also found to reduce decomposition) [18]. After eluting sequentially with 0 , $25 \%$, then $60 \%$ EtOAc/hexanes, fractions containing desired material were then pooled in a round bottom flask, and a very small (ca. 0.1-0.4 mg) crystal of BHT as inhibitor was added, then the solution evaporated. A small quantity of DCM was used to transfer the residue to a small, tared vial, and residual volatiles were removed by repeated coevaporation of the residue with pentanes, followed by high vacuum, to afford $147 \mathrm{mg}$ ( $68 \%$ of theoretical) of the title compound as a clear viscous oil. The material was stored in the freezer in glass vials tightly wrapped with parafilm, and protected from light.

The catechol-methacrylate primer solution was prepared as follows. First, methanol was degassed by sparging with argon for 15-30 min. The primer was removed by gently scraping some of the compound with a spatula, into a tared vial, which was then weighed, fitted with a rubber septa, and purged with argon. Sufficient degassed $\mathrm{MeOH}$ was then added via syringe to make a $1 \mathrm{mg} / \mathrm{mL}$ solution, the septa was then replaced with a screw cap, and the vial was vigorously vortexed for 10-20 min until no further primer could be observed adhering to the sides of the vial. Solutions were sealed tightly, protected from light, and stored in the freezer. 


\subsection{Zirconia Specimen}

The specimens were obtained by cutting presintered zirconia frames: yttria-stabilized tetragonal zirconia polycrystal (Y-TZP, LAVA, Esthetic A3, 3M, St. Paul, MN, USA), using a water cooled diamond saw (Buehler Isomet low speed saw, model \#11-1180, Buehler Ltd., Lake Bluff, IL, USA) with a $0.15 \mathrm{~mm}$ thick diamond blade to $2 \mathrm{~mm}$ thickness and $5 \mathrm{~mm}$ length $\times 5 \mathrm{~mm}$ width. Prior to cutting, the green bodies were presintered in a furnace at $1040{ }^{\circ} \mathrm{C}$ for $2 \mathrm{~h}$. After cutting, all specimens were polished using a custom built polishing machine equipped with $\mathrm{SiC}$ abrasive paper \#1200 in order to have the same degree of surface roughness.

\subsection{Surface Treatment}

We divided specimens into five groups according to the types of used primers. Each group is ZL using Zirconia Liner, AP using Alloy Primer, UP using Universal Primer, CP using fresh Catechol primer $(0.1 \mathrm{mg} / \mathrm{mL})$ in methanol (anhydrous, $99.8 \%$ ) degassed and purged with argon [15] to avoid catecholic auto-oxidation [15], and control (non-treated specimen, negative control). The primers were applied according to the manufacturer's instructions.

\subsection{Surface Morphology}

SEM analysis was conducted for the primed (surface-coated with primers) and the non-primed zirconia surfaces. To prepare the primed samples, the commercial primers were applied on the surfaces as directed in the manuals provided by the manufacturers. Catechol primer solution was applied according to the previous report [15]. The surface images were collected using field emission scanning electron microscope (FESEM, Hitachi S-4700, Hitachi Co., Tokyo, Japan) at two different magnifications $(\times 250, \times 1000)$.

\subsection{Knife-Edge Shear Bond Strength}

Knife-edge shear bond test was conducted based on previous reports $[6,15,19]$ using a modified ISO/TS 11405: 2015 as shown in Figure 2. Briefly, a cured dental resin rod $2.5 \mathrm{~mm}$ in diameter shown in Figure 2 was prepared using a common dental monomer mixture ( $49.5 \mathrm{wt} \%$ of Bis-GMA, $49.5 \mathrm{wt} \%$ of TEGDMA, and $1.0 \mathrm{wt} \%$ of DMAEMA and CQ mixture at 1:2 molar ratio). The bottom of the rod was polished and leveled flat with \#800 SiC abrasive paper to obtain the same surface roughness. Dental resin cement (Bisco, Schaumburg, IL, USA) was then applied onto the bottom surface of the rod (Figure 2c) as to simulate the bottom of the resin. The rod was then placed on the primer-treated zirconia surface at orthogonal to the surface and lightly pushed (Figure 2d). The excess of resin cements at the edge of rods was removed using a dental wax carver to avoid gluing on the side wall of rods prior to the light curing. Subsequently, blue light (Demi ${ }^{\mathrm{TM}}$ Ultra Dental Curing Lights, Kavo Kerr, Orange, CA, USA) was applied all around surface for the complete cure for 20-40 s (Figure 2e). All specimens were stored in water at room temperature for $24 \mathrm{~h}$ prior to bonding strength testing. Knife-edge shear bond test was performed at crosshead down speed of $1 \mathrm{~mm} / \mathrm{min}$ (Figure 2f) by a universal testing machine (UTM 4465, Instron, Norwood, MA, USA). Note that a contribution of the bending moment of the rod was diminished by applying the stress at the bottom surface of the rod (at the interface between substrate and rod). Each test was repeated 10 times with 10 specimens $(n=10)$; average and standard deviation were calculated. 


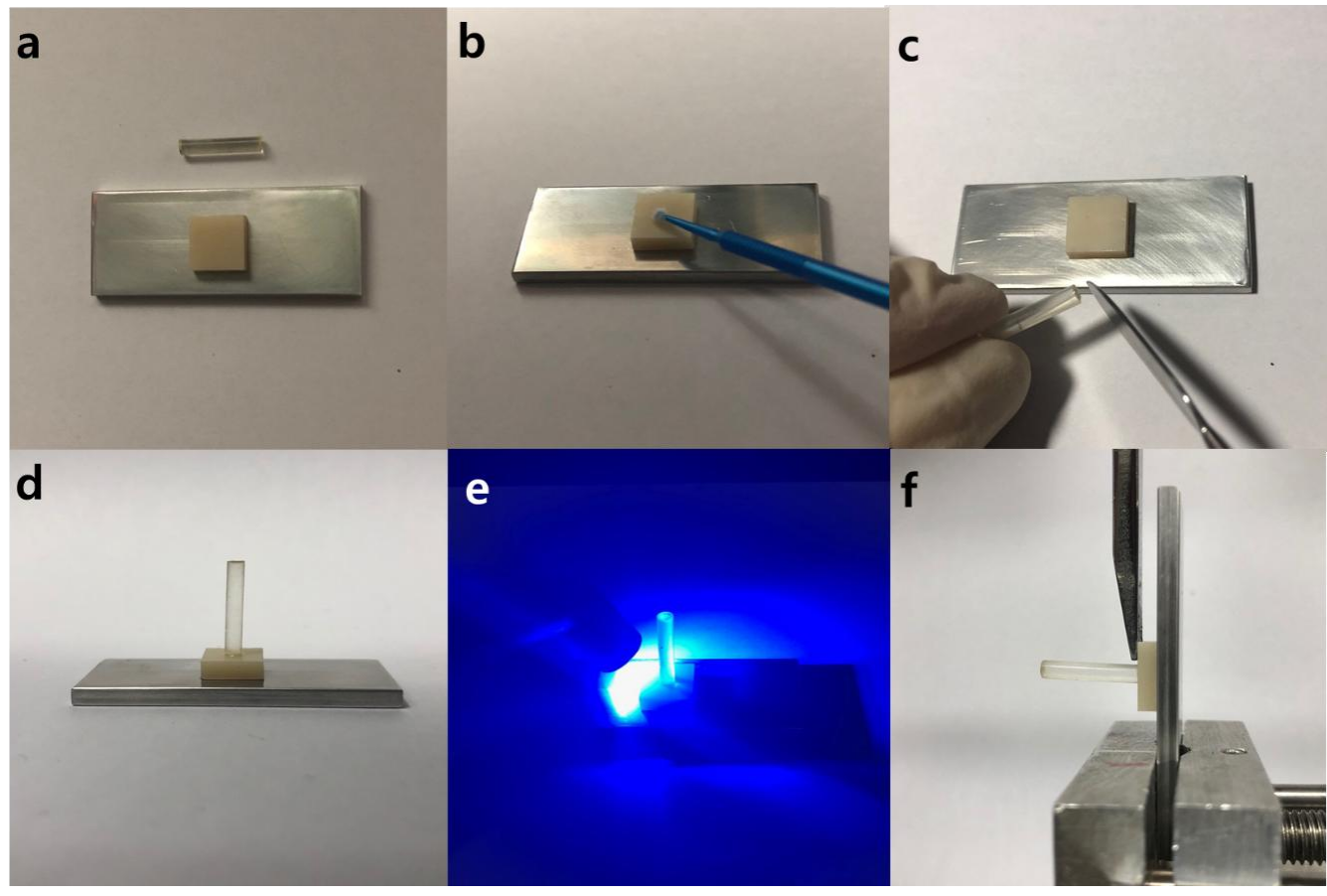

Figure 2. Images taken during sample preparation: (a) a zirconia sample adhered to a stainless-steel plate; (b) apply primer solutions on the zirconia surface; (c) dental resin cement applied to the bottom of resin rod; (d) the resin rod placed on the surface; (e) light curing; (f) knife-edge shear bond test.

\subsection{Statistical Analysis}

Shear bond test data were analyzed using a one-way ANOVA followed by a Tukey's post hoc test at a $\%$ level of significance (SPSS 25.0; SPSS Inc., Chicago, IL, USA).

\section{Results and Discussion}

\subsection{Improved Synthesis of Catechol Methacrylate Primer}

We had some concerns and encountered some difficulties with the previously reported TES-deprotection method [6]. The prior method involved the use of tetrabutylammonium fluoride (TBAF) as limiting reagent, and an excess of the more valuable TES-protected intermediate, the reaction apparently being driven to completion by reaction with adventitious water. Since the prior procedure required that TBAF be used without a buffer, phenolic groups are liable to stay ionized and in the aqueous phase without an acidic workup. Additionally, since the previous conditions are basic due to the use of fluoride anion; catechol moieties are susceptible to autooxidation, resulting in lower yields, yellow sample coloration, and baseline impurities. These basic conditions also apparently are the origin of the observed migration of some of the methacrylates to the internal secondary alcohol, as visible in the proton NMR. In this study, we have increased the yield, purity, and reproducibility of this silyl-deprotection by buffering the TBAF in the reaction mixture with an equimolar amount of benzoic acid (Scheme 1).

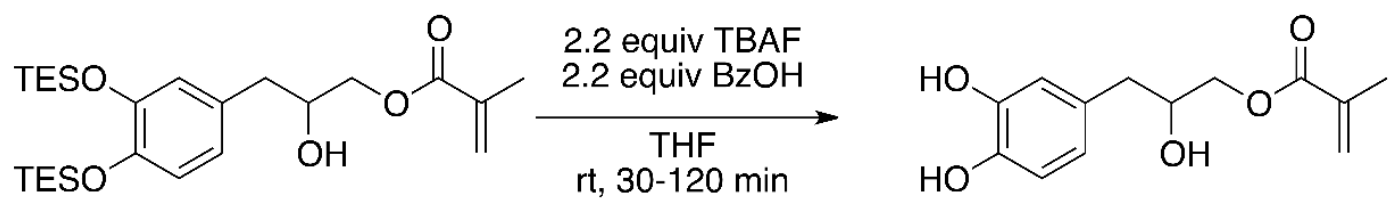

Scheme 1. Synthetic scheme of catechol methacrylate from silylated eugenol. 


\subsection{Scanning Electron Microscope (SEM) Imaging of Primed Zirconia Surface}

Surface morphology of zirconia surfaces with and without primer treatments was studied with a SEM (Figure 3). In the SEM study, CP containing catechol methacrylate shows a complete coverage of zirconia surface, whereas the commercial zirconia primers (ZL, AP, and UP) containing phosphoand/or carboxyl-methacrylates show partial coating on zirconica surfaces. We predicted that higher surface coverage would be positively correlated with the adhesive performance of dental resins to zirconia surface, by allowing for greater microscopic contact areas (reducing microscopic gaps) between zirconia and resin, which was subsequently supported by the results of the shear bond tests (vide infra).

(a) ZL

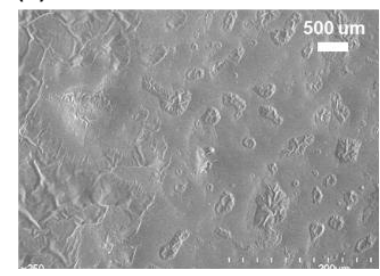

(c) UP

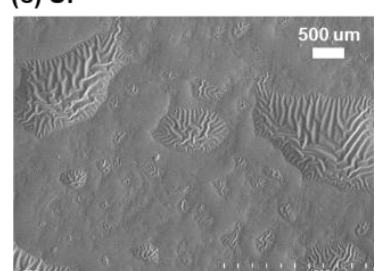

(e) CP (b) AP
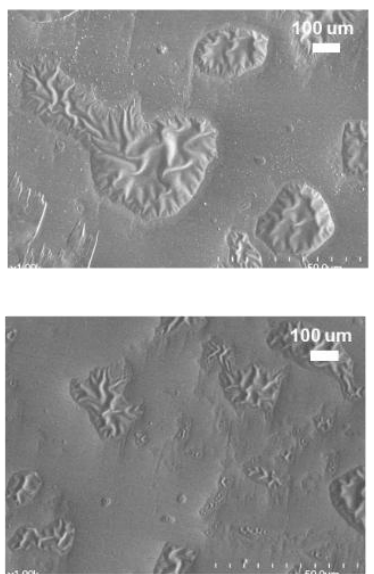

(d) Control

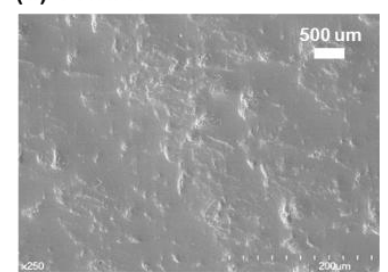

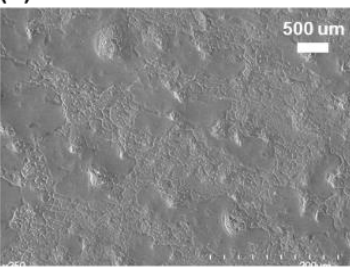
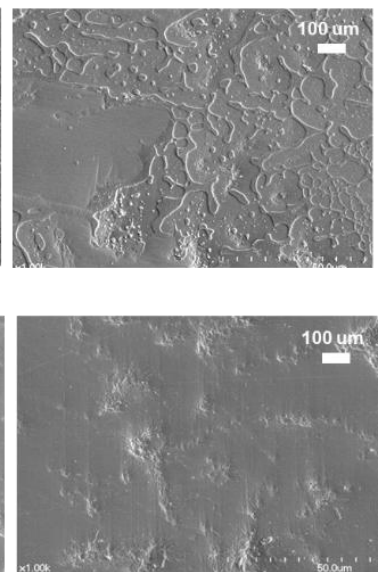
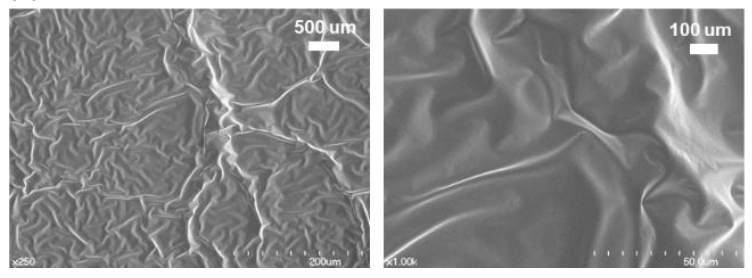

Figure 3. SEM images of zirconia surfaces primed with: (a) ZL: zicornia liner; (b) AP: alloy primer; (c) UP: universal primer; (d) control: no primer; (e) CP: catechol primer.

\subsection{Shear Bond Strength of Dental Adhesives on Bioinspired Catechol Primed Zirconica Surface}

Knife shear bond test was conducted to measure bonding performance of dental adhesives on zirconia surface. Failure mode of each bonding test after the test was monitored by an optical microscope (SZ61, Olympus, Tokyo, Japan), and all the failure occurred on zirconia surface as anticipated. The means, standard deviations of the knife-edge shear bond strengths are shown in Figure 4. With the complete surface coverage of $\mathrm{CP}$ on zirconia surface shown in SEM analysis in Figure 3, the shear bond of $\mathrm{CP}(4.81 \mathrm{MPa} \pm 0.43, n=10$; the mean of maximum shear strength, \pm as standard deviation, and the $\mathrm{n}$ is number of experiments) was higher than commercial zirconia primers containing phosphate and carboxylate methacrylates, i.e., ZL ( $3.52 \mathrm{MPa} \pm 1.01, n=10)$, $\operatorname{AP}(2.61 \mathrm{MPa} \pm 0.76, n=10)$ and $\mathrm{UP}(3.49 \mathrm{MPa} \pm 1.03, n=10)$, and three times stronger than the control without any primer $(1.52 \mathrm{MPa} \pm 0.35, n=10)$. The statistical certainty of the comparison was confirmed by one-way ANOVA with post hoc test. The Tukey's test showed significant differences $(p<0.05)$ between CP (group c in Figure 4) and the each commercial primer (in group a in Figure 4); $p$ value of $\mathrm{CP}$ to ZL was 0.014 , that of $\mathrm{CP}$ to AP was 0.000 and that of $\mathrm{CP}$ to UP was 0.012 . While CP showing significantly higher shear bond strength than group a and group $\mathrm{b}$ (negative control), the Tukey's post hoc analysis indicated that there was a similarity between ZL, AP, and UP (group a) in Figure 4. 


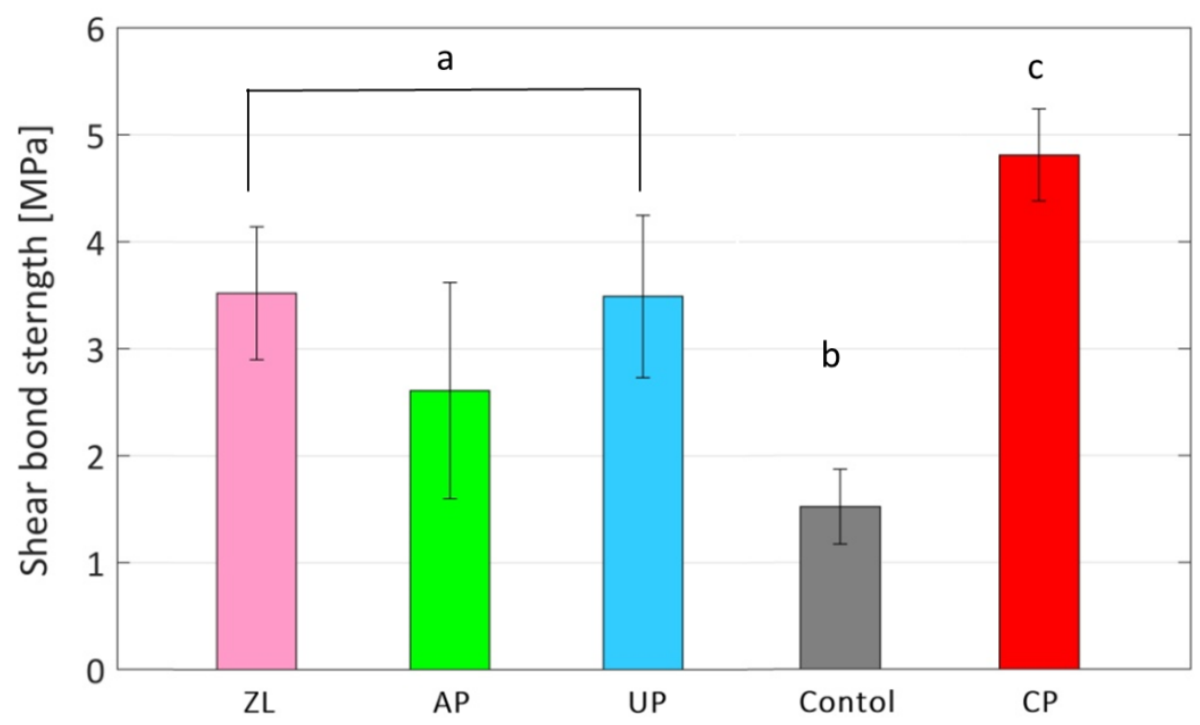

Figure 4. Knife shear bond strength of ZL, AP, UP, control (no primer), and CP.

\section{Conclusions}

In this study, we were able to replicate one of the marine mussel's mechanisms of priming mineral surfaces by using a synthetic small molecule to prime zirconia surfaces. Our strategy of tethering of a polymerizable methacrylate to a catechol moiety was validated by superior bonding performance of our bioinspired primer to zirconia, relative to several popular commercially available formulations. SEM imaging shows that in contrast to those utilizing acidic monomers, the catecholic primer provides a much greater degree of surface coverage, which is correlated with the observed enhancement in dental bonding performance. Considering the catecholic primers' higher strength and ease of application, this priming strategy is well poised for further development in dental applications requiring bonding to zirconia. It would be more interesting if we could have accomplished a rapid underwater curing and more durable underwater adhesion in addition to this strong zirconia bonding that we present here. We will continue to work on this topic of wet-bonding and underwater curing for dental and biomedical applications, and hope this biomimetic priming strategy for stronger dental zirconia bonding encourages other researchers to study and develop better biomimetic adhesives.

Author Contributions: J.A. and K.A. conceived and designed the experiments; M.P. and S.J. performed the experiments; R.L. synthesized the catechol methacrylate; K.A. wrote the manuscript; all authors have contributed to analyzing the data and revising the manuscript.

Funding: This work was supported by the startup funds from the University of Central Florida, National Research Foundation and Marine Biotechnology Program of South Korea (NRF-2018R1A2B6002971 and D11013214H480000110).

Acknowledgments: The authors wish to thank Osaka Organic Chemical Industry Ltd. (Osaka, Japan) for providing the triethylsilyl-protected eugenol methacrylate.

Conflicts of Interest: The authors declare no conflict of interest.

\section{References}

1. Piconi, C.; Maccauro, G. Zirconia as a ceramic biomaterial. Biomaterials 1999, 20, 1-25. [CrossRef]

2. Paes, P.N.G.; Bastian, F.L.; Jardim, P.M. The influence of Y-TZP surface treatment on topography and ceramic/resin cement interfacial fracture toughness. Dent. Mater. 2017, 33, 976-989. [CrossRef] [PubMed]

3. Chuang, S.-F.; Kang, L.-L.; Liu, Y.-C.; Lin, J.-C.; Wang, C.-C.; Chen, H.-M.; Tai, C.-K. Effects of silane- and MDP-based primers application orders on zirconia-resin adhesion-A ToF-SIMS study. Dent. Mater. 2017, 33, 923-933. [CrossRef] [PubMed] 
4. Holten-Andersen, N.; Waite, J.H. Mussel-designed protective coatings for compliant substrates. J. Dent. Res. 2008, 87, 701-709. [CrossRef] [PubMed]

5. Rodriguez, N.R.M.; Das, S.; Kaufman, Y.; Wei, W.; Israelachvili, J.N.; Waite, J.H. Mussel adhesive protein provides cohesive matrix for collagen type-1 $\alpha$. Biomaterials 2015, 51, 51-57. [CrossRef] [PubMed]

6. Seo, S.; Lee, D.W.; Ahn, J.S.; Cunha, K.; Filippidi, E.; Ju, S.W.; Shin, E.; Kim, B.S.; Levine, Z.A.; Lins, R.D.; et al. Significant performance enhancement of polymer resins by bioinspired dynamic bonding. Adv. Mater. 2017, 29, 703026. [CrossRef] [PubMed]

7. Kastrup, C.J.; Nahrendorf, M.; Figueiredo, J.L.; Lee, H.; Kambhampati, S.; Lee, T.; Cho, S.W.; Gorbatov, R.; Iwamoto, Y.; Dang, T.T.; et al. Painting blood vessels and atherosclerotic plaques with an adhesive drug depot. Proc. Nat. Acad. Sci. USA 2012, 109, 21444-21449. [CrossRef] [PubMed]

8. Kivelio, A.; Dekoninck, P.; Perrini, M.; Brubaker, C.E.; Messersmith, P.B.; Mazza, E.; Deprest, J.; Zimmermann, R.; Ehrbar, M.; Ochsenbein-Koelble, N. Mussel mimetic tissue adhesive for fetal membrane repair: Initial in vivo investigation in rabbits. Eur. J. Obstet. Gynecol. Reprod. Biol. 2013, 171, 240-245. [CrossRef] [PubMed]

9. Oh, D.X.; Prajatelistia, E.; Ju, S.-W.; Jeong Kim, H.; Baek, S.-J.; Joon Cha, H.; Ho Jun, S.; Ahn, J.-S.; Soo Hwang, D.S. A rapid, efficient, and facile solution for dental hypersensitivity: The tannin-iron complex. Sci. Rep. 2015, 5, 10884. [CrossRef] [PubMed]

10. Lee, S.-B.; González-Cabezas, C.; Kim, K.-M.; Kim, K.-N.; Kuroda, K. Catechol-functionalized synthetic polymer as a dental adhesive to contaminated dentin surface for a composite restoration. Biomacromolecules 2015, 16, 2265-2275. [CrossRef] [PubMed]

11. Ahn, B.K. Perspectives on mussel-inspired wet adhesion. J. Am. Chem. Soc. 2017, 139, $10166-10171$. [CrossRef] [PubMed]

12. Danner, E.W.; Kan, Y.J.; Hammer, M.U.; Israelachvili, J.N.; Waite, J.H. Adhesion of mussel foot protein Mefp-5 to mica: An underwater superglue. Biochemistry 2012, 51, 6511-6518. [CrossRef] [PubMed]

13. Ahn, B.K.; Das, S.; Linstadt, R.; Kaufman, Y.; Martinez-Rodriguez, N.R.; Mirshafian, R.; Kesselman, E.; Talmon, Y.; Lipshutz, B.H.; Israelachvili, J.N.; et al. High-performance mussel-inspired adhesives of reduced complexity. Nat. Commun. 2015, 6, 8633. [CrossRef] [PubMed]

14. Rickman, R.D.; Verkhoturov, S.V.; Balderas, S.; Bestaoui, N.; Clearfield, A.; Schweikert, E.A. Characterization of surface structure by cluster coincidental ion mass spectrometry. Appl. Surf. Sci. 2004, 231, 106-112. [CrossRef]

15. Shin, E.; Ju, S.W.; An, L.; Ahn, E.; Ahn, J.-S.; Kim, B.-S.; Ahn, B.K. Bioinspired catecholic primers for rigid and ductile dental resin composites. ACS Appl. Mater. Interfaces 2018, 10, 1520-1527. [CrossRef] [PubMed]

16. Ahn, B.K.; Lee, D.W.; Israelachvili, J.N.; Waite, J.H. Surface-initiated self-healing of polymers in aqueous media. Nat. Mater. 2014, 13, 867-872. [CrossRef] [PubMed]

17. Seo, S.; Das, S.; Zalicki, P.J.; Mirshafian, R.; Eisenbach, C.D.; Israelachvili, J.N.; Waite, J.H.; Ahn, B.K. Microphase behavior and enhanced wet-cohesion of synthetic copolyampholytes inspired by a mussel foot protein. J. Am. Chem. Soc. 2015, 137, 9214-9217. [CrossRef] [PubMed]

18. Gelbke, H.P.; Knuppen, R. A new method for preventing oxidative decomposition of catechol estrogens during chromatography. J. Chromatogr. A 1972, 72, 465-471. [CrossRef]

19. Lee, J.-H.; Lee, M.; Kim, K.-N.; Hwang, C.-J. Resin bonding of metal brackets to glazed zirconia with a porcelain primer. Korean J. Orthod. 2015, 45, 299-307. [CrossRef] [PubMed]

(C) 2018 by the authors. Licensee MDPI, Basel, Switzerland. This article is an open access article distributed under the terms and conditions of the Creative Commons Attribution (CC BY) license (http://creativecommons.org/licenses/by/4.0/). 